\title{
ESTUDIO DE LA RELACIÓN ENTRE LA PIEZOMETRÍA Y LA DEFORMACIÓN DEL TERRENO EN EL ACUÍFERO ALMONTE- MARISMAS (ANDALUCÍA, ESPAÑA)
}

\author{
PIEZOMETRIC AND GROUND DEFORMATION RELATIONSHIP AT ALMONTE-MARISMAS AQUIFER \\ (ANDALUCÍA, SPAIN)
}

Miguel González-Jiménez*, Carolina Guardiola-Albert, Héctor Aguilera-Alonso, Marta BéjarPizarro, Gerardo Herrera, Pablo Ezquerro, Juan López-Vinielles, Natalia Fernández, Fernando Ruiz-Bermudo

Instituto Geológico y Minero de España (IGME), C/ Alenza 1, 28003 Madrid, España. miguel.gonzalez@igme.es; c.guardiola@igme.es; h.aguilera@igme.es; m.bejar@igme.es; g.herrera@igme.es; p.ezquerro@igme.es; jlvinielles@gmail.com; n.fernandez@igme.es; f.ruiz@igme.es

\begin{abstract}
:
Groundwater is one of the most important hydric resources of the Spain territory. Nevertheless, heavy groundwater withdrawal generates severe consequences, being land subsidence one of them. The Interferometric Synthetic Aperture Radar (InSAR) technique have been recently exploited to detect and monitor aquifer related deformations. In the present work, relationship between piezometric levels drawdowns and land subsidence has been studied for the higher withdrawals areas within Almonte-Marismas aquifer system (which holds the Doñana Natural Space). Four datasets of radar satellite images, obtained from Sentine-1 satellite, have been processed using the InSAR technique in the period 2014 - 2020. Results show that in some of these areas, like those surrounding El Rocío and Matalascañas villages, the correlation between these two variables is high.
\end{abstract}

Key words: remote sensing, geophysics, GIS, InSAR, hidrogeology, subsidence

Resumen:

El agua subterránea es uno de los recursos hídricos más importantes en el territorio español. La gran cantidad de agua que se retira de ellos puede tener graves consecuencias, entre las que destaca, en algunos acuíferos, la subsidencia del terreno. La técnica de interferometría radar (InSAR), ha sido desarrollada en las últimas décadas para detectar y monitorear las deformaciones relacionadas con los acuíferos. En este trabajo se estudia la posible relación existente entre la variación de los niveles piezométricos y el movimiento del terreno en zonas con grandes extracciones del acuífero Almonte-Marismas (sobre el que se ubica el Espacio Natural de Doñana). Para ello, se han analizado los datos de deformación del terreno obtenidos del satélite Sentinel-1 en el periodo 2014-2020 Los resultados muestran que, en alguna de estas zonas, como las cercanas a las poblaciones de El Rocío y Matalascañas, la correlación entre ambas variables es alta.

Palabras clave: teledetección, geofísica, SIG, InSAR, hidrogeología, subsidencia

\section{Introducción y objetivos}

En las últimas tres décadas, la interferometría con radares de apertura sintética (InSAR) se ha convertido en una importante herramienta de sensor remoto para el estudio temporal y espacial de la deformación del terreno (Massonnet and Feigl 1998; Crosetto et al. 2016). Constituye un conjunto de técnicas eficaces y de bajo coste que permiten estudiar extensas zonas a partir de una alta cantidad de medidas (Tomás et al. 2014). Entre ellas, la técnica de los refractores persistentes (PSI) posibilita estudiar la evolución temporal de la deformación del terreno a partir de la comparación de múltiples imágenes procedentes de radares de apertura sintética (en inglés, SAR).

Una de las múltiples aplicaciones de las técnicas InSAR consiste en el estudio de las deformaciones del terreno generadas por la extracción de agua subterránea. La disminución de la presión que experimentan los poros de la roca cuando el agua es extraída de ellos potencia la consolidación del terreno $\mathrm{y}$, a consecuencia, puede provocar su subsidencia (Ezquerro et al. 2104). Los daños en las infraestructuras civiles son uno de los principales efectos asociados a este fenómeno (Tomás et al. 2005), por lo que la monitorización y el estudio de la deformación del terreno suponen medidas de prevención y mitigación de estos posibles daños (Terzaghi 1925; Ezquerro et al. 2014).

El análisis de la deformación del terreno respecto a las oscilaciones del nivel freático es objeto de estudio en muchas partes del mundo (Galloway et al. 1998; Ezquerro et al. 2014; Bozzano et al. 2015; Béjar-Pizarro et al. 2017; Herrera-García et al. 2021). El presente trabajo tiene la finalidad de desarrollar este tipo de

‘Corresponding Author: Miguel González-Jiménez, miguel.gonzalez@igme.es 
estudios en la zona de Doñana, con el objetivo de analizar cómo las técnicas InSAR podrían mejorar la gestión del agua subterránea y prevenir los riesgos asociados a la subsidencia del terreno.

\section{Zona de estudio}

El acuífero de Almonte-Marismas alberga, en su parte meridional, el Espacio Natural de Doñana (END). El END presenta una extensión de $1030 \mathrm{~km}^{2}(39 \%$ de la superficie del acuífero) y está constituido por un complejo de marismas y lagunas freáticas de gran valor ecológico (Fig. 1). Desde hace varias décadas las aguas subterráneas de Doñana sustentan la mayor parte de las actividades socioeconómicas de la zona, entre las que destacan principalmente la agricultura intensiva y el turismo.

Respecto al turismo, la mayor afluencia de turistas tiene lugar durante las vacaciones de verano e invierno, concentrándose los principales núcleos turísticos en las urbanizaciones de Matalascañas y Mazagón, en el borde suroccidental del acuífero. En el caso de Matalascañas, la alta proximidad (del orden de pocos kilómetros) del área de bombeo a varias lagunas temporales incluidas dentro del END, genera un descenso continuado de los niveles freáticos, así como un fuerte impacto en su actividad ecológica (Serrano and Serrano 1996; DíazPaniagua and Aragonés 2015).

En cuanto a la agricultura intensiva, se trata de una práctica muy común realizada desde 1970 y generalizada en grandes zonas de la superficie del acuífero. Los principales tipos de cultivos son el arroz y los frutos rojos, aunque destacan en menor medida otros como los cítricos. La mayor parte de los arrozales se concentran en las inmediaciones de la localidad de Isla Mayor, situada al este del acuífero y sobre las arcillas de marismas; los cultivos de fresas se concentran en la cabecera de la cuenca del arroyo de La Rocina; mientras que los cítricos lo hacen en las cercanías de El Rocío y Los Hatos, al sur de Villamanrique de la Condesa (Custodio et al. 2009).

Estas actividades requieren de una alta cantidad de agua que, aunque en menor medida para el caso de los arrozales, es obtenida del acuífero de Almonte-Marismas (Custodio et al. 2009). Por esta razón, el estudio se ha focalizado en las zonas de Matalascañas, Isla Mayor, Los Hatos y El Rocío.

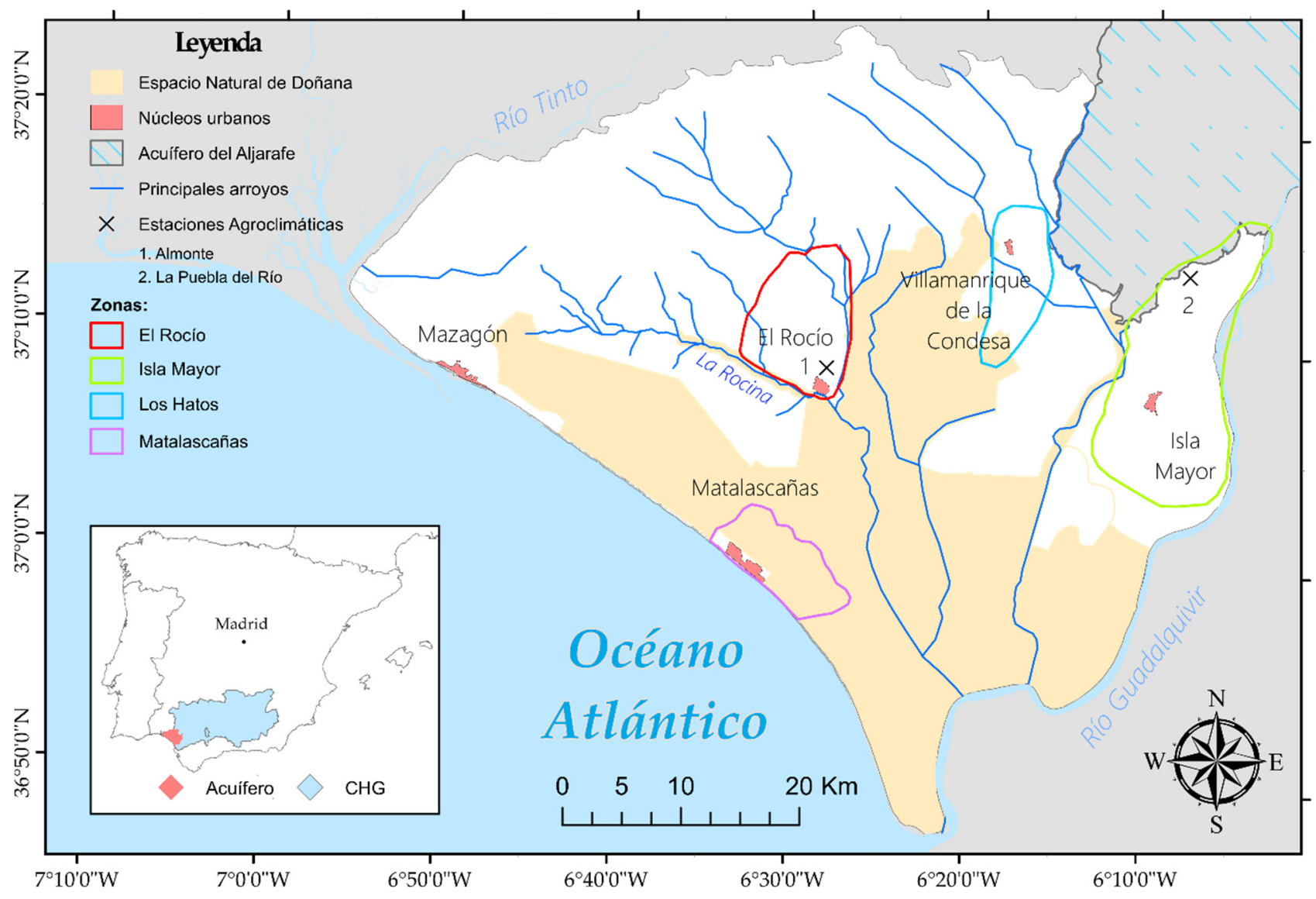

Figura 1: Situación del acuífero Almonte-Marismas y localización de las zonas de estudio, marcadas con polígonos en color.

El acuífero de Almonte-Marismas se encuentra localizado en el suroeste de la Península Ibérica, entre las provincias de Huelva y Sevilla. Pertenece a la cuenca baja del río Guadalquivir, quedando limitado por el río Tinto al oeste, el Guadalquivir al este y el océano Atlántico al sur y suroeste. Presenta una extensión aproximada de $2639 \mathrm{~km}^{2}$ y una topografía suave, encontrándose los puntos más altos (150 msnm) en la parte septentrional y los más bajos (próximos al nivel del mar) en la meridional.

La zona presenta un clima mediterráneo con influencia atlántica, caracterizado por inviernos húmedos y fríos y veranos secos y cálidos. La temperatura y precipitación media anual son de $19^{\circ} \mathrm{C}$ y $550-600 \mathrm{~mm}$ 
respectivamente, presentando esta última una fuerte variación interanual (Manzano and Custodio 2007).

Desde el punto de vista geológico, el acuífero se encuadra en la parte suroccidental de la Depresión del Guadalquivir, quedando limitado por la meseta hercínica en el borde noroccidental y la Cordillera Bética en la arista suroriental. Se trata de un complejo sedimentario formado por arenas y gravas, con frecuentes intercalaciones de arcillas. El muro del sistema acuífero lo constituyen las margas azules del Mioceno Superior, con valores de permeabilidad hidráulica muy bajos.

A grandes rasgos, el sistema acuífero está formado por un acuífero detrítico y libre, y otro confinado bajo los materiales arcillosos y de baja conductividad hidráulica de la marisma. El espesor del acuífero libre aumenta de norte a sur, oscilando desde los $15 \mathrm{~m}$ hasta los $150 \mathrm{~m}$ en la zona de Matalascañas (Guardiola-Albert et al. 2010).

El agua subterránea fluye desde el noroeste hacia el sureste. La recarga media anual es de $200 \mathrm{hm}^{3}$, y se produce de manera principal a través de la precipitación que tiene lugar durante la primavera y el otoño en aquellas zonas en las que afloran los materiales del acuífero libre (Manzano and Custodio 2007). En menor medida, se produce a partir de los excedentes de riego y de la transferencia lateral en la parte nororiental por parte del acuífero de Aljarafe. La descarga del acuífero se produce a través de la salida al mar y por medio del drenaje de los ríos y arroyos, así como por la evapotranspiración y los abundantes bombeos, constituyendo éstos últimos el principal proceso de descarga del acuífero (Manzano and Custodio 2007; Guardiola-Albert et al. 2010).

\section{Datos utilizados}

Para llevar a cabo el estudio se han recopilado, en primer lugar, datos para el periodo 2014-2020 de las dos principales variables de estudio: piezometría y movimiento del terreno. Se ha elegido este lapso temporal por la disponibilidad de los datos de deformación.

Los datos de deformación del terreno consisten en imágenes IW SLC (Interferometric Wide Single Look Complex) procedentes de las trayectorias ascendente (Track 147) y descendente (Track 154) del satélite Sentinel-1. Estas imágenes han sido procesadas a través de la técnica InSAR PSI (Persistent Scatterer Interferometry) y mediante la cadena de procesado PSBAS (Parallel Small BAseline Subset; Manunta et al. 2019), implementada en la plataforma GEP (GeoHazards Exploitation Platform) de la Agencia Espacial Europea (ESA).

De cada una de las trayectorias se han obtenido dos conjuntos de datos para dos periodos de tiempo distintos. El primero abarca desde octubre de 2014 hasta enero de 2020 , mientras que el segundo se extiende entre enero de 2017 y enero de 2020. Cada conjunto de datos consiste en una capa vectorial de entidades puntuales, siendo éstas las que se han utilizado en el análisis de la deformación del terreno (Fig. 2).

En cuanto a la información piezométrica, se han utilizado los piezómetros de la Confederación Hidrográfica del Guadalquivir (CHG) y los sensores del Instituto Geológico y Minero de España (IGME), con un registro de medida mensual y diario, respectivamente (Fig. 3).

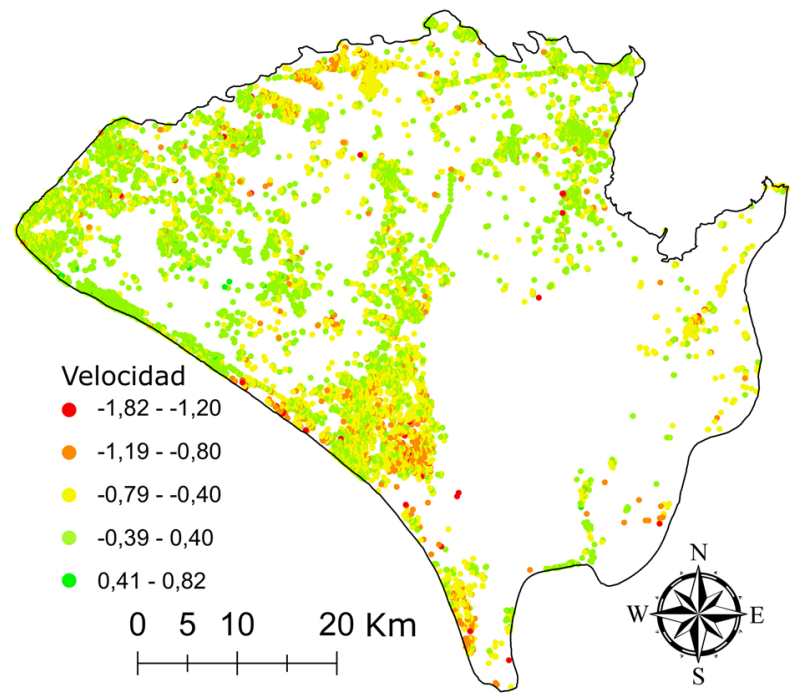

Figura 2: Visualización de todos los puntos SAR del data-set "Descending 2014-2020", representados según la velocidad (cm/año) del movimiento del terreno.

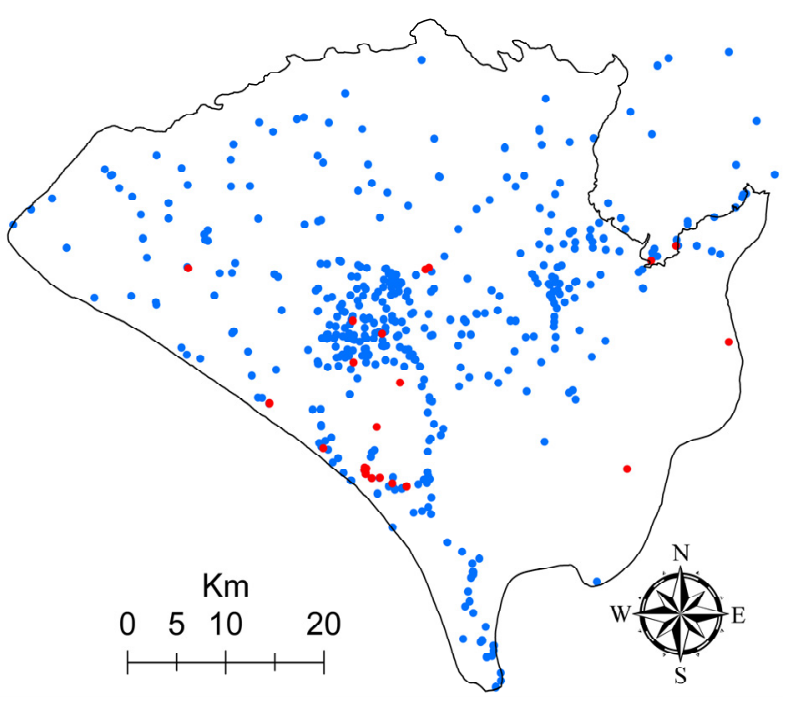

Figura 3: Ubicación de los piezómetros (azules) y sensores (rojos) disponibles en la zona de estudio.

Asimismo, en algunas zonas del área de estudio se han estudiado conjuntamente otras variables que pudieran explicar los resultados obtenidos. Éstas son: las series temporales de precipitación, evapotranspiración (ETP) y extracciones ocurridas en el entorno de Matalascañas. Los datos de extracciones consisten en el registro mensual para el periodo 2014-2020, con valores mensuales típicos generados en base al periodo 20072010, que es el lapso temporal con registro disponible para esta variable.

Los registros históricos de evapotranspiración y precipitación se han obtenido de las estaciones agroclimáticas de 'La Puebla del Río' (para el caso de Isla Mayor) y 'Almonte' (para la zona de Matalascañas), ambas pertenecientes a la Junta de Andalucía (Fig. 1). De la evapotranspiración se han representado tanto los valores originales como la curva de desviación estándar 
acumulada (normalizada a valores entre 0 y 1 ), mientras que de la precipitación únicamente se ha representado la curva de desviación estándar acumulada.

\section{Metodología}

El estudio de la deformación del terreno y su posible relación con la piezometría se ha realizado para cada una de las zonas definidas anteriormente (Fig. 1). Para un estudio más detallado, cada zona ha sido dividida, a su vez, en sub-zonas o agregados en función de la densidad de los puntos SAR. El análisis de la deformación del terreno y la representación gráfica de los resultados se ha realizado para cada agregado.

Este análisis se ha realizado por medio de dos scripts programados en Python, que permiten obtener el promedio de la deformación de cada agregado para cada uno de los conjuntos de datos disponibles, y representarlo gráficamente junto con la evolución de la piezometría de los puntos de agua incluidos en la zona en cuestión, así como otras variables de interés.

Los agregados se han generado mediante la herramienta Aggregate Points, del software ArcMap 10.7, a partir de la cual se han generado entidades poligonales que agrupan los puntos SAR más cercanos. Esta herramienta se ha ejecutado sobre la distribución de los puntos del data-set "Descending 2014 - 2020", por ser el que mayor cantidad y mejor distribuidos presenta los puntos. En el resto de data-sets, la geometría de estos agregados se ha modificado ligeramente con el objetivo de albergar el máximo número de puntos cercanos sin alterar la ubicación e identidad de cada uno de los polígonos.

Una vez constituidos los agregados, se ha procedido a la captura de los puntos incluidos en cada uno de ellos según la zona y el procesado al que pertenezcan. Sobre esta información, almacenada como archivos de texto (formato csv y $t x t$ ), se han ejecutado los scripts.

\section{Resultados y discusión}

Tras el análisis conjunto mediante Python de la piezometría y los datos de deformación del terreno se ha obtenido una imagen de cada uno de los agregados que conforman cada zona. Esta imagen está formada por dos gráficas: una superior y otra inferior. En la primera se encuentran representadas la evolución temporal de la deformación promedio del agregado para cada uno de los procesados con información disponible; mientras que en la segunda se muestra la evolución temporal del nivel freático de los puntos de agua incluidos en la zona de estudio, así como la evolución temporal de alguna variable climática de interés (Fig. 4).

Se considera que existe un posible movimiento vertical del terreno en aquellos agregados en los que, para un mismo momento temporal, la distancia al satélite en la línea de visión (Line of Sight; LOS) varía de manera congruente en ambas trayectorias (ascendente y descendente). Si se cumple este requisito, se podría producir subsidencia cuando la distancia al satélite aumenta respecto a la pasada anterior, y elevación cuando disminuye. Gráficamente esto queda reflejado a través de una correlación directa entre ambas curvas de deformación, tal y como ocurre en el caso de Isla Mayor (Fig. 4).
En general, las curvas de deformación promedio de los agregados de todas las zonas muestran una tendencia subsidente (Fig. 4). La máxima potencial subsidencia acumulada podría alcanzar valores entre 1 y $3,5 \mathrm{~cm}$ para el periodo de estudio $2014-2020$, lo que se traduciría en una tasa de subsidencia anual inferior a $1 \mathrm{~cm}$. La evolución temporal de la deformación del terreno presenta un comportamiento cíclico, oscilando los valores de cada periodo entre 0,5 y $1 \mathrm{~cm}$. De la misma manera, la piezometría muestra un comportamiento cíclico según la tasa de recarga y las extracciones de agua del acuífero. El rango de oscilación de las variaciones piezométricas puede llegar a alcanzar los 6 $\mathrm{m}$.

En la mayor parte de las zonas se observa cierta correlación entre las oscilaciones del nivel freático y la deformación del terreno (Fig. 4). En función de la zona, esta correlación presenta un desfase aproximado de entre 1 y 3 meses, ocurriendo siempre en primer lugar las variaciones en la piezometría, tal y como era de esperar. En las zonas de El Rocío e Isla Mayor, oscilaciones de 1 $\mathrm{m}$ del nivel freático generan movimientos verticales del terreno de aproximadamente $1 \mathrm{~cm}$, mientras que en Los Hatos y Matalascañas, la respuesta del terreno a la variación piezométrica se encuentra mucho más atenuada. En estas dos últimas zonas, variaciones del nivel freático de entre 4 y $6 \mathrm{~m}$ inducen una deformación vertical en el terreno de tan solo $0,5 \mathrm{~cm}$. Esta menor proporcionalidad observada entre la oscilación de la piezometría y la respuesta en la deformación del terreno se puede deber tanto a la profundidad de los niveles freáticos como a la distancia de los pozos de extracción (Ezquerro et al. 2014). También debe de influir el grado de confinamiento del acuífero, siendo esté mayor cuanto mayor sea la potencia de los niveles de arcilla, como ocurre en el caso de Isla Mayor o Los Hatos.

Durante el análisis de la zona de Isla Mayor se ha observado una relación muy interesante entre la piezometría y la ETP. La curva de la desviación estándar acumulada de la ETP presenta una correlación inversa anual (o un desfase de 6 meses) con respecto a la piezometría profunda de la zona. En relación con la serie original de ETP, este desfase es de 3 meses. En esta zona, el acuífero se encuentra confinado por una capa de arcillas de hasta 50 metros de potencia, por lo que resulta difícil que las oscilaciones del nivel freático se encuentren generadas por variaciones estacionales. En base al estudio de Van der Kamp y Maathuis (1991), podría considerarse que estas variaciones del nivel freático profundo son consecuencia de las fluctuaciones en la carga mecánica que ejerce la humedad sobre el acuífero (Van der Kamp and Schmidt 1997), aunque sería necesario realizar un análisis más exhaustivo de esta zona para poder establecer esta relación con seguridad.

La tendencia general observada en las señales del movimiento vertical del terreno podría indicar cierta componente inelástica para todas las zonas acuífero. El coeficiente de elasticidad de un acuífero depende de la naturaleza litológica del mismo, siendo este menor cuanto mayor sea la cantidad de materiales arcillosos y de baja permeabilidad que contenga. En acuíferos detríticos como el de la cuenca de Madrid (Ezquerro et al. 2014), el coeficiente de elasticidad es muy alto, lo que genera que las elevaciones y subsidencias provocadas por la recarga y descarga del acuífero respectivamente, 
presenten una magnitud muy similar. Por esta razón y, según las conclusiones del trabajo de Ezquerro et al. (2014), la ligera proporcionalidad observada entre los ascensos y descensos del terreno en todas las zonas estudiadas del acuífero Almonte-Marismas, así como la constante tendencia decreciente de la señal InSAR, determina una naturaleza elasto-plástica para el mismo.

Aunque en las gráficas resultantes se observa la existencia de una correlación entre las variables del estudio, se recomienda realizar un tratamiento estadístico de las mismas para corroborar las correlaciones observadas. Una de las técnicas más utilizadas para realizar este análisis es mediante las herramientas Wavelet (Grinsted et al. 2004; Tomás et al. 2016) que, a través del análisis en el dominio frecuencial y temporal de las series temporales, permiten identificar las periodicidaes principales de las variables del estudio, así como las posibles correlaciones entre ellas.
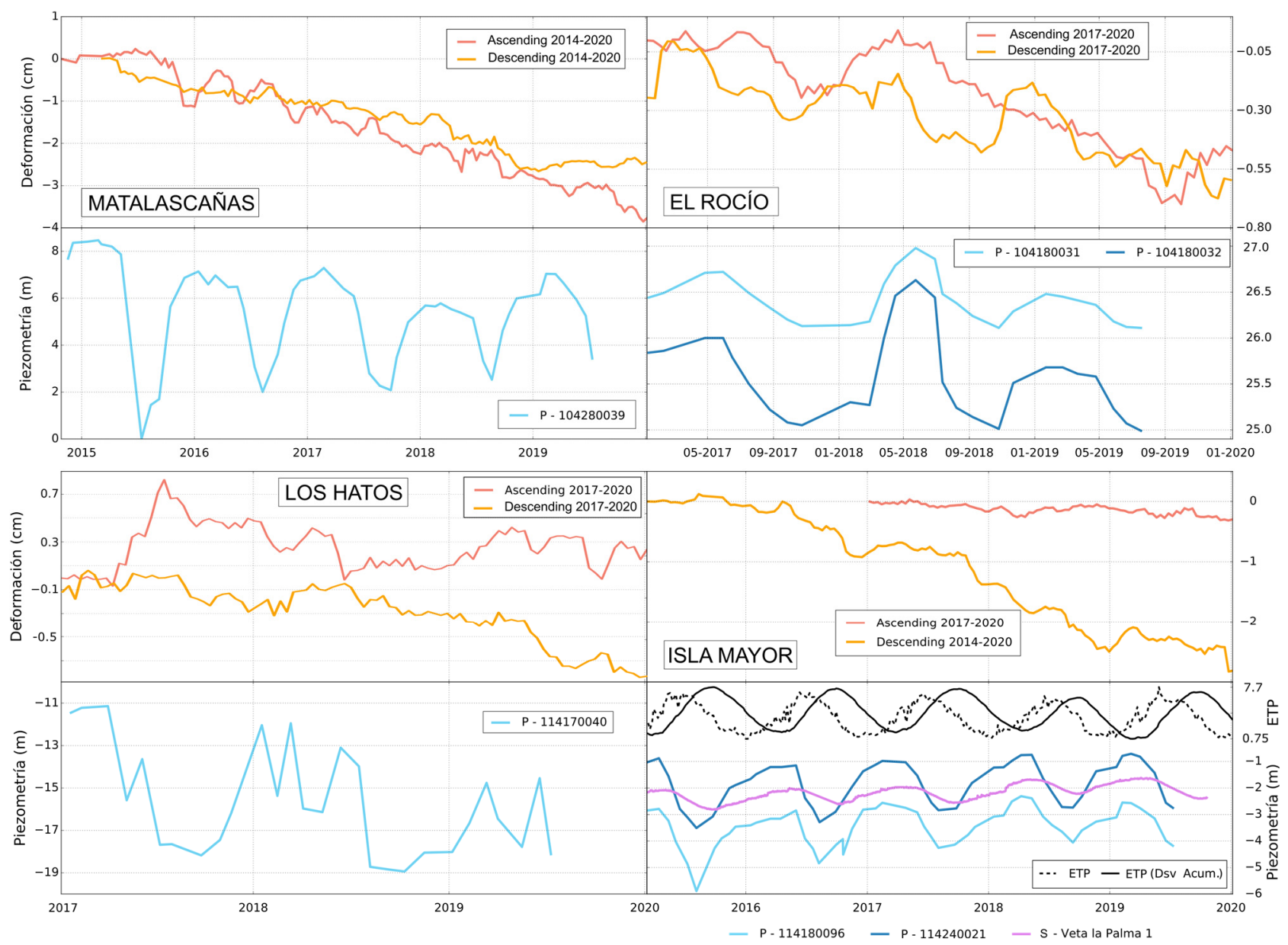

Figura 4: Gráficas representativas de la relación existente entre la piezometría (curvas azules) y la deformación del terreno (curvas naranjas y rojas) para cada una de las zonas analizadas. En color negro se muestran los valores reales de evapotransipiración (ETP; línea discontinua) y la curva de la desviación acumulada de la evapotranspiración (Dsv. Acum.; línea continua) normalizada al intervalo $0-1$.

\section{Conclusiones}

En este trabajo se ha analizado la posible relación existente entre la piezometría y la deformación del terreno mediante técnicas InSAR y a través de la confección de dos scripts con Python y el uso de softwares de sistemas de información geográfica. Tras el análisis de un total de cuatro zonas (Isla Mayor, El Rocío, Los Hatos y Matalascañas) se alcanzan dos conclusiones. En primer lugar, toda la zona podría encontrarse sometida a una subsidencia constante y paulatina de aproximadamente $1 \mathrm{~cm} / a n ̃ o$, que refleja la naturaleza elasto-plástica del acuífero. $Y$, en segundo lugar, todas las zonas muestran cierta correlación entre la deformación del terreno, obtenida de la señal InSAR, y la oscilación de la piezometría. Para estudiar en profundidad esta correlación, convendría aplicar técnicas estadísticas, como podrían ser las herramientas Wavelet.

\section{Agradecimientos}

Este trabajo es parte de las actividades subvencionadas dentro del Sistema Nacional de Garantía Juvenil (PEJ2018-002477), financiado por la Fundación Tripartita para la Formación en el Empleo, la iniciativa YEI (Youth Employment Initiative) y el Fondo Social Europeo (FSE).

Los datos Copernicus Sentinel-1 se obtuvieron y procesaron en la plataforma GEP de la ESA en el marco del GEP Early Adopters Programme.

Agradecemos al IGME y a la CHG por proporcionar los datos de piezometría, así como a la Junta de Andalucía por suministrar los datos meteorológicos. 


\section{References}

BÉJAR-PIZARRO, M., EZQUERRO, P., HERRERA, G., TOMÁS, R., GUARDIOLA-ALBERT, C., HERNÁNDEZ, J. M. R., FERNÁNDEZ-MERODO, J.A., MARCHAMALO, M., and MARTÍNEZ, R., 2017. Mapping groundwater level and aquifer storage variations from InSAR measurements in the Madrid aquifer, Central Spain. Journal of Hydrology, 547, pp. 678689. DOI: 10.1016/j.jhydrol.2017.02.011

BOZZANO, F., ESPOSITO, C., FRANCHI, S., MAZZANTI, P., PERISSIN, D., ROCCA, A., and ROMANO, E., 2015. Understanding the subsidence process of a quaternary plain by combining geological and hydrogeological modelling with satellite InSAR data: The Acque Albule Plain case study. Remote Sensing of Environment, 168, pp. 219-238. DOI: 10.1016/j.rse.2015.07.010

CROSETTO, M., MONSERRAT, O., CUEVAS-GONZÁLEZ, M., DEVANTHÉRY, N., and CRIPPA, B., 2016. Persistent scatterer interferometry: A review. ISPRS Journal of Photogrammetry and Remote Sensing, 115, pp. 78-89 DOI: 10.1016/j.isprsjprs.2015.10.011

CUSTODIO, E., MANZANO, M., and MONTES, C., 2009. Las aguas subterráneas en Doñana: aspectos ecológicos y sociales. Consejería de medio Ambiente. 249 pages

DÍAZ-PANIAGUA, C., and ARAGONÉS, D., 2015. Permanent and temporary ponds in Doñana National Park (SW Spain) are threatened by desiccation. Limnetica, 34, pp. 407-424. DOI: 10.23818/limn.34.31

EZQUERRO, P., HERRERA, G., MARChAMALO, M., TOMÁS, R., BÉJAR-PIZARRO, M., and MARTíNEZ, R., 2014. A quasi-elastic aquifer deformational behavior: Madrid aquifer case study. Journal of Hydrology, 519, pp. 1192-1204. DOI: $10.1016 / j$.jhydrol.2014.08.040

GALlOWAY, D. L., HUDNUT, K. W., INGEBRITSEN, S. E., PHILLIPS, S. P., PELTZER, G., ROGEZ, F., and ROSEN, P. A., 1998. Detection of aquifer system compaction and land subsidence using interferometric synthetic aperture radar, Antelope Valley, Mojave Desert, California. Water Resources Research, 34(10), pp. 2573-2585. DOI: 10.1029/98WR01285

GRINSTED, A., MOORE, J. C., and JEVREJEVA, S., 2004. Application of the cross wavelet transform and wavelet coherence to geophysical time series. Nonlinear Processes Geophys, European Geoscience Union (EGU). 11(5/6), pp. 561-566.

GUARDIOLA-ALBERT, C., GARCÍA-BRAVO, N., MEDIAVILLA, C., and MARTÍN-MACHUCA, M., 2010. Gestión de los recursos hídricos subterráneos en el entorno de Doñana con el apoyo del modelo matemático del acuífero AlmonteMarismas. Boletín Geológico y Minero, 120(3), pp. 361-376.

HERRERA-GARCÍA, G., EZQUERRO, P., TOMÁS, R., BÉJAR-PIZARRO, M., LÓPEZ-VINIELLES, J., ROSSI, M., MATEOS, R.M., CARREÓN-FREYRE, D., LAMBERT. J., TEATINI, P., CABRAL-CANO, E., ERKENS, G., GALLOWAY, D., HUNG, WEI-CHIA, KAKAR, N., SNEED, M., TOSI, L., WANG, H., and YE, S., 2021. Mapping the global threat of land subsidence. Science, 371(6524), pp. 34-36 DOI: 10.1126/science.abb8549

MANUNTA, M., DE LUCA, C., ZINNO I., CASU, F., MANZO, M., BONANO, M., FUSCO, A., PEPE, A., ONORATO, G., BERARDINO, P., DE MARTINO, P., and LANARI, R., 2019. The Parallel SBAS Approach for Sentinel-1 Interferometric Wide Swath Deformation Time-Series Generation: Algorithm Description and Products Quality Assessment. IEEE Transactions on Geoscience and Remote Sensing, 57(9), pp. 6259-6281.

MANZANO, M., and CUSTODIO, E., 2007. Las aguas subterráneas en Doñana y su valor ecológico. Enseñanza de las Ciencias de la Tierra, 15(3), pp. 305-316.

MASSONNET, D., and FEIGL, K., 1998. Radar interferometry and its application to changes in the earth's surface. Reviews of geophysics, 36, pp. 441-500. DOI: 0.1029/97RG03139

SERRANO, L., and SERRANO L., 1996. Influence of groundwater exploitation for urban water supply on temporary ponds from the Donana National Park (SW Spain). Journal of Environmental Management, 46(3), pp. 229-238. DOI: 10.1006/jema.1996.0018

TERZAGHI, K., 1925. Settlement and Consolidation of Clay. McGraw-Hill, New York, pp. 874-878.

TOMÁS, R., LI, Z., LOPEZ-SÁNCHEZ, J. M., LIU, P., and SINGLETON, A., 2016. Using wavelet tools to analyse seasonal variations from InSAR time-series data: a case study of the Huangtupo landslide. Landslides, 13(3), pp. 437-450. DOI: 10.1007/s10346-015-0589-y

TOMÁS, R., MÁRQUEZ, Y., LOPEZ-SÁNCHEZ, J. M., DELGADO, J., BLANCO, P., MALLORQUÍ, J. J., MARTÍNEZ, M., HERRERA, G., and MULAS, J., 2005. Mapping ground subsidence induced by aquifer overexploitation using advanced Differential SAR Interferometry: Vega Media of the Segura River (SE Spain) case study. Remote Sensing of Environment, 98(2-3), pp. 269-283. DOI: 10.1016/j.rse.2005.08.003

TOMÁS, R., ROMERO, R., MULAS, J., MARTURIÀ, J. J., MALLORQUÍ, J. J., LOPEZ-SANCHEZ, J. M., HERRERA, G., GUTIÉRREZ, F., GONZÁlEZ, P.J., FERNÁNDEZ, J., DUQUE, S., CONCHA-DIMAS, A., COCKSLEY, G., CASTAÑEDA, C., CARRASCO, D., and BLANCO, P., 2014. Radar interferometry techniques for the study of ground 
subsidence phenomena: a review of practical issues through cases in Spain. Environmental earth sciences, 71(1), pp. 163-181.DOI: 10.1007/s12665-013-2422-z

VAN DER KAMP, G., and MAATHUIS, H., 1991. Annual fluctuations of groundwater levels as a result of loading by surface moisture. Journal of hydrology, 127, pp. 137-152. DOI: 10.1016/0022-1694(91)90112-U

VAN DER KAMP, G., and SCHMIDT, R., 1997. Monitoring of total soil moisture on a scale of hectares using groundwater piezometers. Geophysical Research Letters, 24(6), pp. 719-722. DOI: 10.1029/97GL00521 